\title{
To frame or not to frame? Effects of message framing and risk priming on mouth rinse use and intention in an adult population- based sample
}

\author{
Gert-Jan de Bruijn ${ }^{1}$ (D)
}

Received: March 6, 2018/Accepted: September 10, 2018/Published online: September 21, 2018

(C) The Author(s) 2018

\begin{abstract}
Good dental hygiene is key to public health. To promote dental hygiene behaviours, persuasive messages are key. Message framing is a popular theory that has seen mixed evidence. In this web-based experimental study, interaction effects of message frame, behavioural function, and risk priming were investigated on mouth rinse use and intentions in a representative sample of Dutch adults. Final included sample contained 549 participants $(50.1 \%$ male, mean age $=47.4, \mathrm{SD}=16.1)$ and outcomes were immediate mouth rinse product choice, intentions to use mouth rinse, and mouth rinse behaviour at 2-week follow-up. Results demonstrated a theorized significant interaction between message frame and behavioural function were mouth rinse product choice. Two-week follow-up mouth rinse behaviour was affected by an interaction between message frame and risk prime. Message framing can thus be employed to promote dental health, but it requires attention to moderation effects which are inconsistent across behavioural outcomes.
\end{abstract}

Keywords Message framing - Persuasive communication · Dental health · Adults · Intention · Behaviour

Gert-Jan de Bruijn

g.j.debruijn@uva.nl

1 Amsterdam School of Communication Research ASCoR, University of Amsterdam, PO Box 15804, $1001 \mathrm{NH}$ Amsterdam, The Netherlands

\section{Introduction}

Good dental health is protective of various physical and mental ailments (Allen, 2003; Griffin et al., 2012; Mattila et al., 1989), but a substantial proportion of adults have insufficient levels of dental hygiene behaviours to obtain oral health related benefits (Sheiham, 2005). The use of mouth rinse leads to reduced caries increment (Marinho et al., 2016) and lower dental plague scores (Van Leeuwen et al., 2017) and should be stimulated using effective persuasive messages (Tonetti et al., 2015). One of the most popular persuasive strategies is message framing (Rothman \& Salovey, 1997), where people are presented with either positive consequences of action or the negative consequences of inaction. Depending on the behavioural function (prevention or detection of illness), either a gain-frame (for preventive action) or a loss-frame (for detection behaviour) is theorized to be more persuasive. The idea behind the interaction between message frame and behavioural function is rooted in prospect theory (Kahneman \& Tversky, 1979), whereby preferences for one of two choices are different, depending upon how these outcomes are framed, even though the factual outcomes are equal. When outcomes are framed in terms of gains, people become risk-avoidant and prefer the low-risk choice option, while risk-seeking choice options are preferred when outcomes are framed as losses. Thus, message framing assumes an important role for risk and behavioural function in modifying choice preferences following message frame exposure (Rothman \& Salovey, 1997). Although most health behaviours serve only one behavioural function, mouth rinse can be communicated as having either detection or prevention functions (Rothman et al., 1999). 
The issue of risk of a moderator of framing effects has some evidence in the dental health domain. Although main effects of message frame are often absent (Gallagher \& Updegraff, 2012; Updegraff et al., 2007, 2015) interactions with avoidance-approach motivation, whereby people are motivated to either avoid negative outcomes or obtain positive outcomes, (Mann et al., 2004; Updegraff et al., 2007) and perceived risk to dental health problems (Updegraff et al., 2015) are commonly found. For instance, in a study on dental flossing in American adults, Updegraff et al. (2015) found that gain-framed messages were more persuasive when people perceived themselves to be a low risk for dental health problems, but loss-framed messages were more persuasive when people perceived to be at high risk for dental health problems. Outside the realm of dental health, however, there is little support that risk is a consistent moderator of message framing effects (Covey, 2015; Van 't Riet et al., 2014).

One reason for this lack of moderation is that most studies on risk and message framing have relied on selfreports on risk. However, optimistic biases lead to personal and self-reported risk assessments that are often substantially lower than statistical estimates would suggest (Weinstein, 1987; Weinstein \& Klein, 1995). One way to overcome these biases is by implicitly activating risk, for instance via priming (Erb et al., 2002; Herr, 1986; Janiszewski \& Wyer, 2014). Priming refers to the activation of the accessibility of mental representations that subsequently influence behaviour outside conscious awareness (Bargh \& Chartrand, 2000) and priming effects have been found for illness schemas (Henderson et al., 2007), risk (Erb et al., 2002; Gilad \& Kliger, 2008), and risk communication studies (Pavey \& Sparks, 2012). For instance, in a study on risk versus no-risk choices, Erb et al. (2002) primed participants to be either risk-seeking or risk-avoidant and, in a subsequent unrelated choice task, those who were primed to be risk-seeking were more likely to opt for the risky choice in that unrelated task. In contrast, those primed to be risk-avoidant opted more often for the no-risk choice. Further, less than $3 \%$ of the participants indicated that their choice was influenced by the previous priming task. Thus, risk-priming techniques can unconsciously influence behavioural choices that differ in their level of risk. Although these findings have intuitive appeal for risk moderation in message framing work, no research has investigated how risk priming affects message framing effects. One of the purposes of the present research was to investigate how risk priming affects message framing effects.

Message framing effects have mostly been investigated for linear interval outcomes, such as duration (i.e. numbers of minutes of vigorous activity) or frequency (i.e. number of days per week on which one has performed dental hygiene behaviours) of action, or on Likert-scale type questions such as intention and attitudes ( $\mathrm{Li}$ et al., 2014; Updegraff et al., 2015). Given its roots in prospect theory, such outcomes are potentially problematic, as prospect theory tenets have primarily been investigated for dichotomous choices that vary in (un)certainty (Kahneman \& Tversky, 1979). In fact, outside the realm of (dental) health, stronger framing effects have been found for response modes that are construed as distinct choices than for response modes that are construed as judgments or rating on evaluation scales (Kühberger, 1998). Furthermore, most of the evidence on message framing effects on these different response modes is derived from studies were distinct choices and scale judgments were not studied within the same sample, thus prohibiting a direct intraindividual comparison of framing effects on those response modes. Another purpose of the present research was to investigate message framing effects on the aforementioned two different response modes within the same sample.

To summarize, the present study investigated the effects of message frame, risk priming and behavioural function on mouth rinse use and intentions, as well as a dichotomous choice outcome, where one mouth rinse product had to be selected from a set of two products that differed in behavioural function (prevention or detection). Following message framing theory, it was hypothesized that a significant interaction would emerge between message frame and behavioural function such that loss-framed message (versus gain-framed messages) would be more persuasive when the mouth rinse served a detection (versus prevention) function and that these effects would be more pronounced for dichotomous choices than for scale judgments. It was also hypothesized that a significant interaction would emerge between risk prime and message frame, such that loss-framed messages (versus gain-framed messages) would be more persuasive for participants who were primed to be risk seeking (versus risk averse). Finally, it was hypothesized that a three-way interaction would emerge, such that the combination of loss-framed messages with detection function (versus gain-framed messages with prevention function) would be even more persuasive when participants were primed to be risk seeking (versus risk averse).

\section{Method}

\section{Participants and procedures}

Participants were Dutch adults who were registered members of an ISO-certified Internet Research Panel company. The panel consists of over 16,000 individuals and is representative for the Dutch population in terms of age, gen- 
der, and educational attainment, and was actualised no longer than 12 months prior to data collection. The panel applies a 'double-active-opt-in' approach, meaning that panel members are actively aware of their voluntary membership. Participation in individual studies is voluntary, but panel members cannot provide preferences to be included or excluded for specific types of research. Participation in the present study was rewarded with 190 credit points, with a monetary value of $€ 2,11$. Inclusion criteria for the present study were adult age and not wearing dentures-no other study criteria were made. The Institutional Review Board approved the study protocol. Eligible adults were invited to participate in a study on dental health and those who agreed to participate were forwarded to an online survey. In this survey, they provided information on how often they had used mouth rinse in the previous month. They also provided information on their perception of their susceptibility to caries, dental plaque, gingivitis, and a bad breath in case of irregular dental hygiene, and indicated the perceived severity of these consequences. These two constructs were presented in random order to the participants. Within each construct, items were also presented in random order.

Following this, participants were randomly assigned to a scrambled sentence task (SST) that primed either a riskseeking or a risk-avoidant mind-set. After completing the SST, participants were then randomly allocated to one of four messages that were a function of both type of behavioural function (detection or prevention) and message frame (gain or loss). Participants were instructed to carefully read their allocated message. After reading the persuasive message, participants then entered the next phase, where they were presented with two types of bottles of mouth rinse on a single page. Each bottle had either a prevention function or a detection function and could be placed in the upper or lower half of the page. Across participants, the place where each of these bottles appeared was randomized, so that half of the participants saw the prevention (or detection) mouth rinse bottle in the upper half and the detection (or prevention) bottle in the lower half. Prior the accessing the product choice page, participants were instructed to select one of these two mouth rinse bottles, but no further instruction (i.e. select the first product that they immediately felt most inclined to select) or reason (i.e. you will enter a raffle where you can win the product of your choice) was given. After choosing, participants were asked to indicate on a separate page how certain $(-3=$ very unsure; $+3=$ very sure $)$ they were of their choice. Finally, participants indicated their intention to use mouth rinse in the next 2 weeks. An online debriefing questioned participants about their presumed link between the SST (masked as a language proficiency task) and the mouth rinse manipulations and questions. If participants gave hints of a link between the SST and the mouth rinse manipulations, they were further requested to elaborate on the link between the two. Two weeks after, participants provided data on mouth rinse use in the previous 2 weeks, as well as their intention to use mouth rinse in the next 4 weeks. Unique respondent numbers were used to match the longitudinal data.

\section{Flow of participants}

Over 1500 participants $\left(\mathrm{N}=1556, M_{\text {age }}=48.9, S D=\right.$ 15.8, 805 males $(51.7 \%)$ provided baseline data. There were no differences in baseline variables across the eight experimental conditions (all $F \mathrm{~s}<1.75$, all $p \mathrm{~s}>.094$ ) and so randomization was considered successful. Of the 1556 baseline participants, eight participants were excluded because they indicated that the SST was meant to prime something $(n=5)$ or to prime risk $(n=3)$. No participants were excluded for not constructing grammatically correct sentences. Of the remaining 1548, a further 604 (58.7\%) were excluded because the time they spent on the message page was either too short ( $<20 \mathrm{~s}, n=586$ ) or too long (zscore $>2, n=18$ ). Of the remaining 944 participants, 305 participants were additionally excluded, because they did not regard the persuasive message as conveying negative consequences of not using mouth rinse (loss-framed, $n=175$ ) or positive consequences of using mouth rinse (gain-framed, $n=130$ ). This exclusion was done to ascertain that included participants individually perceived the framed messages as intended, a strategy that has precedent in message framing work in dental health (10). ${ }^{1}$ Successful manipulation did not depend on main and interaction effects of conditions (all $p s>.248$ ). Of the remaining 639 participants, a further 90 participants did not respond to the follow-up email, leaving a final sample of 549 participants. Dropout was unrelated to baseline variables and message evaluations (all $p s>.433$ ) and to main and interaction effects of conditions (all $p$ s $>.408$ ). The flow of participants is graphically presented in Fig. 1.

\section{Measures}

Mouth rinse use was assessed at baseline and at follow-up and participants indicated how frequent $(0=$ never, 7 = every day of the week) they had used mouth rinse in the past month (baseline) and in the past 2 weeks (followup). Because of the manipulation of risk, two common

\footnotetext{
${ }^{1}$ When compared with the total sample, participants who passed the manipulation check perceived the persuasive message more as intended (mean scores) and were homogeneous about their perception (smaller standard deviation), gain-framed: $\mathrm{M}=2.1, \mathrm{SD}=0.6$ versus $\mathrm{M}=0.8, \quad \mathrm{SD}=1.7 ; \quad$ loss-framed $\mathrm{M}=-1.9, \quad \mathrm{SD}=0.6 M=0.0$, $S D=1.8$.
} 


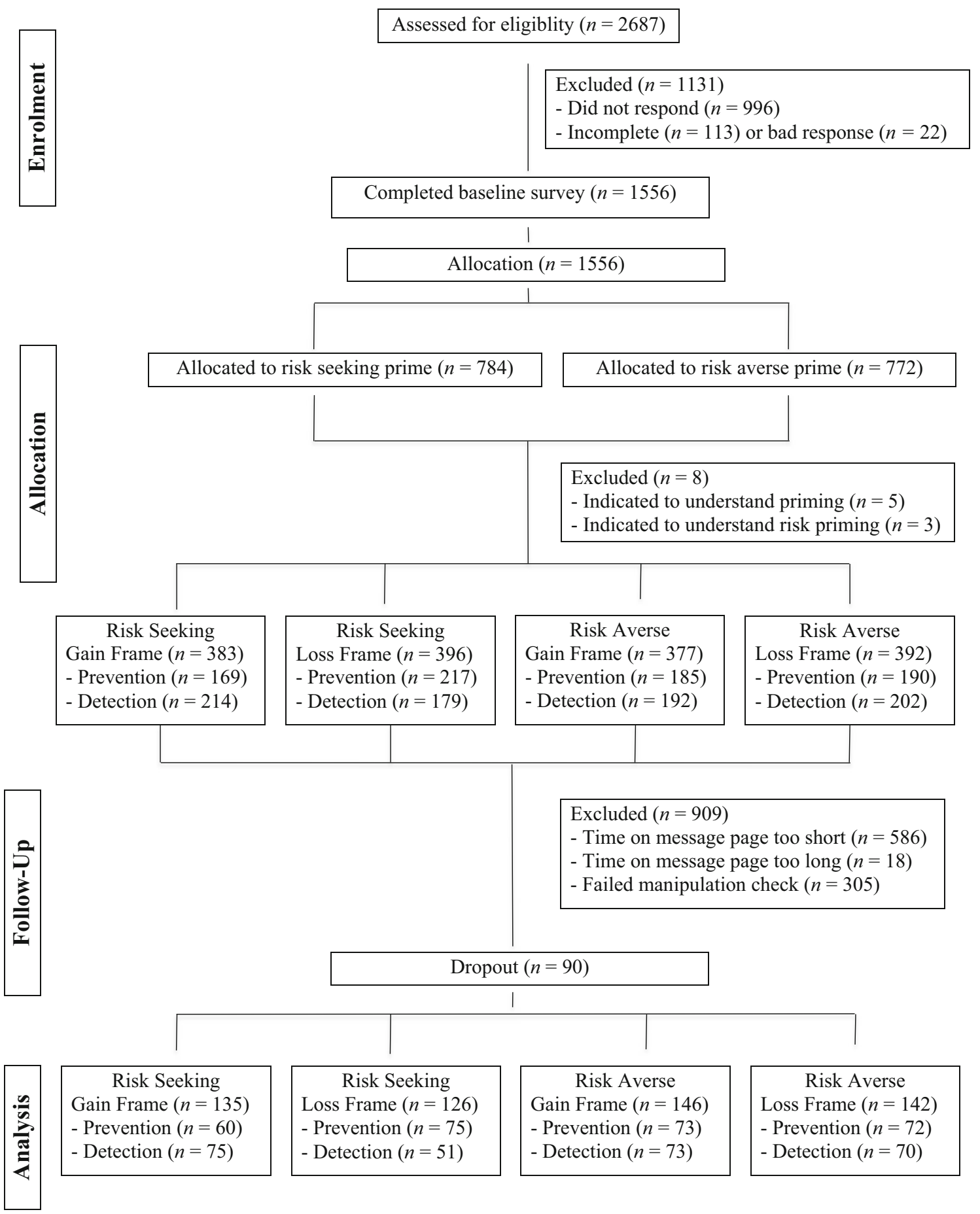

Fig. 1 Flowchart

indicators of risk (i.e. severity and susceptibility) were a priori determined to be included as covariates and assessed at baseline. For these items, suggestions from Protection Motivation Theory (Rogers \& Prentice-Dunn, 1997) and the Health Belief Model (Janz \& Becker, 1984) were followed. Severity of oral diseases was assessed with five items $(\alpha=.96)$ that followed the stem 'If I do not perform dental hygiene behaviours (such as mouth rinse use) daily, the negative consequences will be' (1) severe, (2) serious, (3) considerable, (4) large, and (5) not to be underestimated. Susceptibility for oral disease was assessed with four items $(\alpha=.81)$ that followed the stem 'If I do not 
perform dental hygiene behaviours (such as mouth rinse use) daily, I will suffer from' (1) caries, (2) dental plaque, (3) gingivitis, and (4) a bad breath. Intention to use mouth rinse was assessed after experimental manipulation with the item 'I intend to use mouth rinse daily'. For the immediate follow-up intention measurement, the reference period 'in the next 2 weeks' was used, whereas the reference period 'in the next month' was used for the 2-week follow-up intention measurement. Items for multi-item constructs were randomized and scores for constructs were averaged. For choice of mouth rinse, two advertisements were made to reflect professional, yet fake, mouth rinse products (see Fig. 2). Those advertisements emphasized either the product's detection or prevention functions.

\section{Manipulations}

For the priming of risk, an SST was used that was introduced to participants as a language proficiency test. The SST was chosen, because research has indicated that such a supraliminal task leads to strong and relatively long priming effects (Bargh \& Chartrand, 2000). Participants received twelve sets of five words each, from which a sentence had to be made with four of these five words. In each sentence, one word thus had to be dropped in order to construct a grammatically correct sentence. Eight of these 12 sentences were constructed to either prime risk seeking or risk avoidance-the remaining four sentences were fillers. The five-word sentences were presented in random order and were checked for proper grammar by the author. All participants created grammatically correct sentences. "Appendix 1" has the full list of the words used for the SST.

To manipulate both type of frame and type of behaviour, four separate messages were constructed and pretested based on previous oral hygiene message framing studies (Pakpour et al., 2013; Rothman et al., 1999; Updegraff et al., 2007, 2015). Type of behaviour was manipulated by presenting the mouth rinse information as having either a prevention or a detection function. Type of frame was manipulated by emphasizing the gains (or losses) of using (loss-frame: not using) mouth rinse in the persuasive text and message heading. Word count for the messages in the detection condition was 216 (gain-framed) or 220 (lossframed) words. In the prevention condition, word count was either 170 (gain-framed) or 174 (loss-framed) words. Each of the four messages had four separate paragraphs in Calibri font (size 14) and an eight-word heading in Calibri bold font (size 17). The full text and the layout of these messages are in "Appendix 2". The time participants spent on this message page was assessed by hidden timers. A pretest revealed that minimum reading time of each of these messages was $20 \mathrm{~s}$.

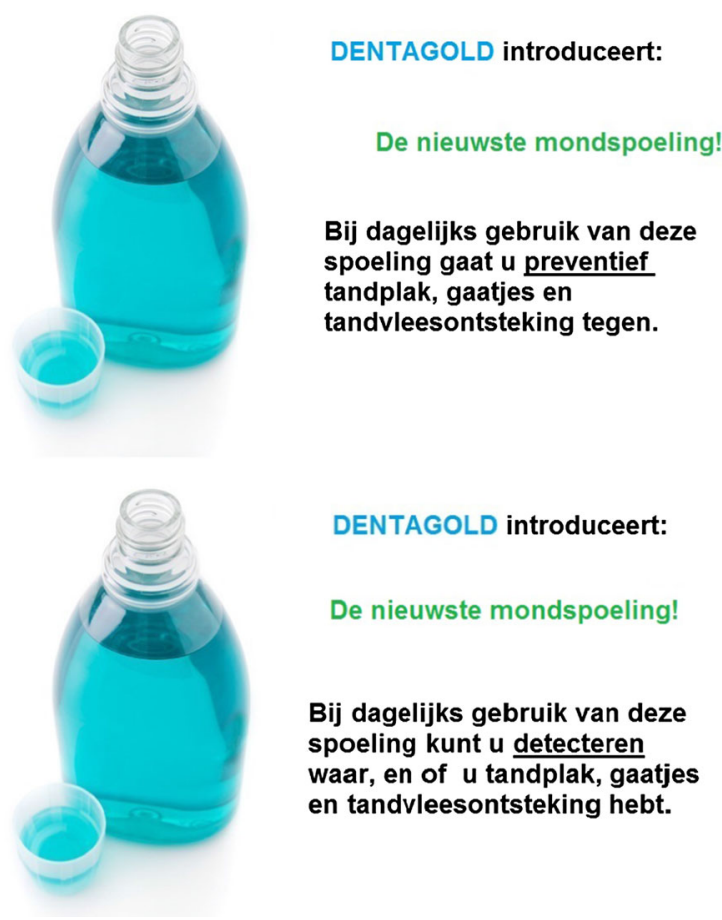

Dentagold introduces

The newest mouth rinse

When used daily, this mouth rinse will help you prevent caries, dental plagues, and gingivitis

Fig. 2 Mouth Rinse Products. Note. Participants were exposed to Dutch version. The English translation of the main text is on the right 
After message exposure, a manipulation check was performed where participants indicated whether the message they had just read highlighted either positive consequences or negative consequences of performing (lossframe: not performing) dental hygiene behaviours. $(-3=$ very negative consequences; $+3=$ very positive consequences). Finally, because message evaluations may potentially explain message framing effects on dental health (Rothman et al., 1999; Updegraff et al., 2007), message evaluations were also assessed. Participants indicated whether or not $(-3=$ totally disagree; +3 totally agree) they felt the message they had just read was (presented in random order to participants) (1) credible, (2) personally relevant, (3) informative, (4) interesting, (5), irritating, (6) ridiculous, (7) worth remembering, (8) useful, and (9) exaggerated. A single factor was extracted and so a mean score was computed $(\alpha=.93)$, with a higher score indicating a more positive message evaluation.

\section{Statistical analyses}

The main and interactive effects of type of message frame, type of behavioural function, and type of risk priming on product choice were tested using stepwise logistic regression analysis. For this logistic regression, choice certainty, message evaluation and baseline severity and susceptibility were entered as covariates. For immediate follow-up intention, product choice (detection or prevention product) was entered as an additional covariate. For the effects on the 2-week follow-up outcome measures, product choice and immediate follow-up intention were additional covariates. Thus, the number of covariates ranged from 4 (baseline product choice) to 6 (2-week follow-up outcomes).

For the logistic regression analysis, covariates were entered first, after which main effects of conditions were entered in the second block. The two-way interactions were entered thereafter, and the three-way interaction was included in the fourth step. For the immediate follow-up intention outcome, a 2 (type of priming: risk seeking vs. risk averse) $\times 2$ (type of message: gain-framed vs. lossframed) $\times 2$ (type of behaviour: detection vs prevention) ANCOVA was performed. For the 2-week follow-up outcomes, a 2 (type of priming: risk seeking vs. risk averse) $\times 2$ (type of message: gain-framed vs. lossframed) $\times 2$ (type of behaviour: detection vs prevention) MANCOVA was used. For the follow-up analyses, a MANOVA power analysis using $G^{*}$ Power software (Faul et al., 2007) showed that a minimum sample of 468 was needed to detect a small-to-medium effect size difference in MANOVA $\left(.17^{2}=.03\right)$ for 8 groups, 6 predictors (covariates), and two response variables (2-week follow-up intention and behaviour) with $\alpha$ set at .05 and power at .95 .
The effect size $\eta^{2}$ was used to qualify multivariate effects (small: $\quad \eta^{2}>=.02 ; \quad$ medium: $\eta^{2}>=.13 ; \quad$ large: $\eta^{2}>=.26$ ). Significant multivariate effects were followed up by univariate tests and planned comparisons, where Cohen's $d$ (1992) was the effect size estimate (small: $d>.30$; medium: $d>.50$; large: $d>.80$ ). Earlier interpretations for effect sizes for significant odds ratios were also followed (Chen et al., 2010).

\section{Results}

\section{Participants}

Table 1 has the descriptives for baseline characteristics, message evaluation, and dependent variables for the total analysed sample (Table 1) at baseline and at the 2-week follow-up. Table 1 shows that, of the 639 included baseline participants, $311(48.7 \%)$ were male and average age was 47.5 years $(S D=16.0$, range $18-87$ years). Little over a quarter $(n=173,27.1 \%)$ had low educational background, and little over ten percent $(n=66,10.3 \%)$ of the participants reported to use mouth rinse daily and over half $(n=376,58.8 \%)$ reported to never use mouth rinse. Participants reported that the negative consequences of not performing dental health behaviours were relatively severe and that they were susceptible to those negative consequences. In general, message evaluation was relatively positive and there was an effect of type of message frame, $F(1,638)=31.75, p<.001, \eta^{2}=.05$, on message evaluation, such that message evaluations were higher for gainframed messages $(\mathrm{M}=1.20, \mathrm{SE}=.06)$ than for lossframed messages $(\mathrm{M}=0.72$, $\mathrm{SE}=.06)$. Therefore, message evaluation was entered as a covariate to study effects on persuasion outcomes. ${ }^{2}$

\section{Effects on mouth rinse choice}

Of the 639 participants included at the baseline assessment, 191 (29.9\%) opted for the detection mouth rinse. On average for both products, participants were relatively certain of their choice $(M=1.56, S D=1.31)$ with choice certainty above midscale for the majority of the sample ( $n=536,83.9 \%)$. Choice certainty was higher for the preventive mouth rinse $(M=1.61, S E=.06)$ than for the detection mouth rinse $(M=1.19, S E=.12), \quad F \quad(1$, $623)=9.95, p=.002, \eta^{2}=.02$. Choice certainty was also influenced by the interaction of mouth rinse choice and

\footnotetext{
${ }^{2}$ Participants rated the message that portrayed mouth rinse as having a detection function equally credible, exaggerated, and ridiculous, as the message that portrayed it as a prevention function. This effect did not depend upon previous mouth rinse use, $F<1$. (I thank two anonymous reviewers for bringing this up).
} 
Table 1 Proportions and means and standard deviations for baseline characteristics, message evaluation, and immediate and 2-week follow-up measures for analysed sample

\begin{tabular}{|c|c|c|c|c|c|c|c|c|c|}
\hline & \multirow[t]{4}{*}{ Total } & \multicolumn{8}{|c|}{ Risk priming } \\
\hline & & \multicolumn{4}{|c|}{$\begin{array}{l}\text { Risk seeking } \\
\text { Behavioural function }\end{array}$} & \multicolumn{4}{|c|}{$\begin{array}{l}\text { Risk averse } \\
\text { Behavioural function }\end{array}$} \\
\hline & & \multicolumn{2}{|c|}{$\begin{array}{l}\text { Prevention } \\
\text { Message frame }\end{array}$} & \multicolumn{2}{|c|}{$\begin{array}{l}\text { Detection } \\
\text { Message frame }\end{array}$} & \multicolumn{2}{|c|}{$\begin{array}{l}\text { Prevention } \\
\text { Message frame }\end{array}$} & \multicolumn{2}{|c|}{$\begin{array}{l}\text { Detection } \\
\text { Message frame }\end{array}$} \\
\hline & & Gain & Loss & Gain & Loss & Gain & Loss & Gain & Loss \\
\hline \multicolumn{10}{|l|}{ Baseline } \\
\hline Sample size & $n=639$ & $n=68$ & $n=90$ & $n=91$ & $n=67$ & $n=82$ & $n=79$ & $n=81$ & $n=81$ \\
\hline$\%$ Male & 48.7 & 48.5 & 50.0 & 48.4 & 35.8 & 48.8 & 55.7 & 45.7 & 54.3 \\
\hline \multirow[t]{2}{*}{$\%$ Education } & $27.1 / 41.5$ & $25.0 / 42.6$ & $16.7 / 41.1$ & $27.5 / 40.7$ & $34.3 / 35.8$ & $29.3 / 41.5$ & $21.5 / 53.2$ & $32.1 / 37.0$ & $32.1 / 39.5$ \\
\hline & $M(S D)$ & $M(S D)$ & $M(S D)$ & $M(S D)$ & $M(S D)$ & $M(S D)$ & $M(S D)$ & $M(S D)$ & $M(S D)$ \\
\hline Age & $47.5(16.0)$ & $47.0(15.8)$ & $46.8(15.9)$ & $47.7(16.0)$ & $47.3(18.4)$ & $50.1(15.8)$ & $46.4(15.9)$ & $48.5(14.4)$ & $46.3(16.6)$ \\
\hline T0 mouth rinse & $1.3(2.3)$ & $1.1(2.2)$ & $1.0(2.0)$ & $0.9(1.9)$ & $1.5(2.5)$ & $1.8(2.6)$ & $1.4(2.5)$ & $1.4(2.3)$ & $1.2(2.1)$ \\
\hline T0 severity & $1.0(1.5)$ & $1.1(1.5)$ & $0.9(1.6)$ & $1.1(1.4)$ & $1.1(1.5)$ & $1.2(1.4)$ & $0.9(1.4)$ & $1.1(1.6)$ & $1.0(1.4)$ \\
\hline T0 susceptibility & $1.9(1.0)$ & $1.9(0.9)$ & $1.7(1.4)$ & $1.9(1.0)$ & $1.9(0.9)$ & $1.9(1.0)$ & $1.8(1.1)$ & $2.0(1.0)$ & $1.7(1.0)$ \\
\hline \multicolumn{10}{|l|}{ Immediate follow-up } \\
\hline$\%$ Preventive mouth rinse & 70.1 & 91.2 & 80.0 & 53.8 & 61.2 & 87.8 & 82.3 & 53.1 & 54.3 \\
\hline Choice certainty & $1.6(1.3)$ & $1.8(1.1)$ & $1.6(1.3)$ & $1.5(1.2)$ & $1.3(1.5)$ & $1.7(1.4)$ & $1.8(1.3)$ & $1.4(1.3)$ & $1.5(1.5)$ \\
\hline T1 message evaluation & $1.0(1.1)$ & $1.0(1.0)$ & $0.6(1.1)$ & $1.2(0.8)$ & $0.7(1.1)$ & $1.3(1.1)$ & $0.7(1.1)$ & $1.3(1.1)$ & $0.9(1.7)$ \\
\hline $\mathrm{T} 1$ mouth rinse intent & $-0.3(2.1)$ & $-0.2(1.9)$ & $-0.7(2.0)$ & $-0.4(1.9)$ & $-0.2(2.1)$ & $0.3(2.3)$ & $-0.2(2.2)$ & $-0.3(2.1)$ & $-0.6(1.9)$ \\
\hline \multicolumn{10}{|l|}{ Two-week follow-up } \\
\hline Sample size & $n=549$ & $n=60$ & $n=75$ & $n=75$ & $n=51$ & $n=73$ & $n=72$ & $n=73$ & $n=70$ \\
\hline $\mathrm{T} 2$ mouth rinse intent & $-0.5(2.2)$ & $-0.8(2.0)$ & $-0.7(2.2)$ & $-1.0(2.0)$ & $-0.4(2.4)$ & $-0.6(2.2)$ & $-0.0(2.2)$ & $0.1(2.5)$ & $-0.5(2.1)$ \\
\hline $\mathrm{T} 2$ mouth rinse use & $1.4(2.4)$ & $1.1(2.1)$ & $1.2(2.3)$ & $0.8(1.8)$ & $1.7(2.6)$ & $1.4(2.3)$ & $1.8(2.6)$ & $2.2(2.8)$ & $1.1(2.1)$ \\
\hline
\end{tabular}

$\mathrm{T} 0$ reflects baseline assessment, T1 reflects assessment immediately after follow-up, T2 reflects 2-week follow-up assessment. Education reflects $\%$ low (left) and middle (right) education. Intent, severity, susceptibility, choice certainty, and message evaluation variables could range from -3 (totally disagree) to +3 (totally agree). Mouth rinse is in days per week

behavioural function, $F(1,623)=15.64, \quad p<.001$, $\eta^{2}=.02$. In the prevention function condition, choice certainty was much stronger for the preventive mouth rinse $(M=1.84, S E=.08)$ than the detection mouth rinse $(M=0.90, S E=.20)$. In the detection message condition, differences between the preventive mouth rinse $(M=1.38$, $S E=.10)$ and the detection mouth rinse $(M=1.48, S E=$ .11) were smaller. Although no other effects on choice certainty were identified (all $F$ s $<1$ ), choice certainty was entered as an additional covariate for the logistic regression analysis.

Table 2 shows the results of this stepwise logistic regression analysis. After controlling for the covariates, the addition of the experimental factors showed a large effect of behavioural function, $O R=4.35$, 95\% CI [3.00-6.37], $p<.0001$. Participants in the detection function condition were more likely to select the detection mouth rinse over the prevention mouth rinse $(74.9 \%)$ than participants in the prevention function condition were to select the preventive mouth rinse over the detection mouth rinse $(60.5 \%), \chi^{2}(1$, $n=639)=66.97, p<.001$. The next step showed an additional two-way interaction between type of message frame and type of behavioural function, $O R=2.32,95 \%$ CI [1.05-5.13], $p=.037$. This odds-ratio was of medium effect size (29) and follow-up tests showed that participants were more than two times more likely to select a preventive mouth rinse when a prevention message was paired with a gain-frame, $O R=7.28,95 \%$ CI [4.00-13.25], $p<.001$, than paired with a loss-frame message, $O R=$ $3.17,95 \%$ CI [1.92-5.25], $p<.001$. This is corroborated by the finding that in the prevention function conditions, participants were more likely to select the preventive mouth rinse product when its prevention function was paired with a gain-framed message than with a loss-frame message, $O R=1.96,95 \%$ CI [1.03-3.73], $p=.042$. However, message frame did not impact choice in the detection function condition, $O R=0.85, \quad 95 \% \quad \mathrm{CI}$ [0.55-1.33], $p=.479$. Finally, no other two-way interactions were identified on product choice, nor was there a significant three-way interaction. 
Table 2 Results from stepwise logistic regression analysis for mouth rinse choice

\begin{tabular}{|c|c|c|c|}
\hline & OR & $95 \% \mathrm{CI}$ & $p$ value \\
\hline \multicolumn{4}{|l|}{ Step 1} \\
\hline Type of prime & 1.08 & {$[0.75-1.55]$} & 678 \\
\hline Type of behaviour & 4.35 & {$[3.00-6.37]$} & $<.001$ \\
\hline Type of frame & 1.24 & {$[0.85-1.80]$} & .265 \\
\hline \multicolumn{4}{|l|}{ Step 2} \\
\hline Type of prime & 1.19 & {$[0.65-2.19]$} & .567 \\
\hline Type of behaviour & 3.18 & {$[1.67-6.03]$} & $<.001$ \\
\hline Type of frame & 0.95 & {$[0.53-1.70]$} & .863 \\
\hline Prime $*$ behaviour & 0.88 & {$[0.40-1.91]$} & .732 \\
\hline Frame $*$ behaviour & 2.32 & {$[1.05-5.13]$} & .037 \\
\hline Frame $*$ prime & 0.95 & {$[0.45-2.01]$} & .899 \\
\hline \multicolumn{4}{|l|}{ Step 3} \\
\hline Type of prime & 1.37 & {$[0.70-2.67]$} & .362 \\
\hline Type of behaviour & 3.72 & {$[1.79-7.75]$} & $<.001$ \\
\hline Type of frame & 1.07 & {$[0.57-2.01]$} & .836 \\
\hline Prime $*$ behaviour & 0.64 & {$[0.23-1.78]$} & .388 \\
\hline Frame $*$ behaviour & 1.62 & {$[0.55-4.81]$} & .382 \\
\hline Frame $*$ prime & 0.75 & {$[0.30-1.85]$} & .526 \\
\hline Prime $*$ behaviour $*$ prime & 2.15 & {$[0.43-10.72]$} & .352 \\
\hline
\end{tabular}

$O R$ odds ratio, $C I$ confidence interval. Mouth rinse choice was coded $0=$ detection mouth rinse, $1=$ prevention mouth rinse. Type of prime was coded $0=$ risk averse; $1=$ risk seeking. Type of behaviour was coded $0=$ detection; $1=$ prevention. Type of frame was coded $0=$ loss frame; 1 = gain frame. Choice certainty, message evaluation, and baseline severity and susceptibility are added as covariates

\section{Effects on intentions and behaviour}

Table 3 has the test statistics for the multivariate effects on intention and behaviour, whilst Figs. 3, 4 and 5 depict the estimated means and standard errors for these dependents variables for each of the two-way interactions. For intention to use mouth rinse immediately after experimental manipulation, there was a significant interaction between behavioural function and type of prime, with follow-up tests revealing that intention differences between detection $(M=-0.44, \quad S E=.17, \quad n=126) \quad$ and prevention $(M=-0.37, \mathrm{SE}=.16, n=135)$ were not as pronounced $d=0.03,95 \%$ CI [- 0.28-0.21] as in the risk-seeking prime condition than in the risk-averse prime condition (detection: $\quad M=-0.65, \quad S E=.16, \quad n=143$; prevention: $M=0.08, \quad S E=.15, \quad n=145, \quad d=0.39, \quad 95 \% \quad$ CI [0.16-0.63]).

For the 2-week follow-up outcomes, there was a multivariate effect of message frame, Wilks' $\Lambda=0.983, F$ (2, 536) $=4.57, p=.011, \eta^{2}=.02$, and an interaction between risk priming and message frame, Wilks' $\Lambda=0.988, F(2,536)=3.26, p=.039, \eta^{2}=.01$. Test of between-subject effects showed that this multivariate effect was related to a main effect of message frame for mouth rinse intentions and an interaction effect of risk priming and message frame for mouth rinse use (see Table 3). For the main effect of message frame, planned comparisons showed that intentions were less negative in the lossframed message condition $(M=-0.31, S E=.08$, $n=268)$ than in the gain-framed message condition $(M=-0.68, \quad S E=.08, \quad n=281), \quad d=0.27, \quad 95 \% \quad \mathrm{CI}$ [0.10-0.44]. For the interaction, planned comparisons showed that, in the risk-seeking condition, mouth rinse use was higher for participants who were exposed to the lossframed message $(M=1.57, S E=.16, n=126)$ than for those exposed to the gain-framed message $(M=0.99$, $S E=.15, n=135), d=0.33,95 \%$ CI [0.08-0.57]. In the risk-avoidant condition, differences between the lossframed $(M=1.44, S E=.15, n=146)$ and gain-framed condition $(M=1.60 S E=.14, n=142)$ were less pronounced, $d=0.09,95 \%$ CI $[-0.32$ to 0.14$]$. No other multivariate effects of conditions were found (all $F_{\mathrm{s}}>$ 2.38, all $p \mathrm{~s}>.093)$.

\section{Discussion}

In the present study, effects of message framing and risk priming on immediate dichotomous product choice and intentions, as well as on 2-week follow-up intention and behaviour regarding mouth rinse were investigated in a large sample of Dutch adults using the tenets of message 
Table 3 Mean squares, F-values, statistical significance, and eta-squared for mouth rinse use intentions at immediate and 2-week follow-up and mouth rinse use at 2-week follow-up

\begin{tabular}{|c|c|c|c|c|}
\hline & Mean square & $F$-value & $p$ value & $\eta^{2}$ \\
\hline \multicolumn{5}{|l|}{ Immediate follow-up intention } \\
\hline Type of risk prime & 0.76 & 0.23 & 635 & .00 \\
\hline Type of behavioural function & 11.96 & 3.55 & .060 & .01 \\
\hline Type of message frame & 1.40 & 0.41 & .520 & .00 \\
\hline Risk prime $\times$ behavioural function & 13.74 & 4.08 & .044 & .01 \\
\hline Risk prime $\times$ message frame & 0.47 & 0.14 & .708 & .00 \\
\hline Behavioural function $\times$ message frame & 6.29 & 1.87 & .172 & .00 \\
\hline Risk prime $\times$ behavioural function $\times$ message frame & 3.63 & 1.08 & .299 & .00 \\
\hline \multicolumn{5}{|l|}{ Two-week follow-up intention } \\
\hline Type of risk prime & 8.15 & 4.25 & $.040^{\#}$ & .01 \\
\hline Type of behavioural function & 0.01 & .004 & .950 & .00 \\
\hline Type of message frame & 17.54 & 9.15 & .003 & .02 \\
\hline Risk prime $\times$ behavioural function & 0.53 & 0.28 & .599 & .00 \\
\hline Risk prime $\times$ message frame & 1.37 & 0.71 & .399 & .00 \\
\hline Behavioural function $\times$ message frame & 0.16 & 0.09 & .770 & .00 \\
\hline Risk prime $\times$ behavioural function $\times$ message frame & 0.72 & 0.38 & .539 & .00 \\
\hline \multicolumn{5}{|l|}{ Two-week follow-up behaviour } \\
\hline Type of risk prime & 7.81 & 2.66 & .103 & .01 \\
\hline Type of behavioural function & 0.01 & 0.00 & .950 & .00 \\
\hline Type of message frame & 5.69 & 1.94 & .164 & .00 \\
\hline Risk prime $\times$ behavioural function & 1.27 & 0.42 & .510 & .00 \\
\hline Risk prime $\times$ message frame & 18.59 & 6.34 & .012 & .01 \\
\hline Behavioural function $\times$ message frame & 0.42 & 0.14 & .705 & .00 \\
\hline Risk prime $\times$ behavioural function $\times$ message frame & 0.19 & 0.06 & .801 & .00 \\
\hline
\end{tabular}

Values are assessed at $d f(1,627)$ for immediate follow-up outcome and at $d f(2,536)$ for 2-week follow-up outcomes. Message evaluation and baseline severity and susceptibility, and product choice are entered as covariates for immediate intention measure, with immediate intention as an additional covariate for the 2 -week follow-up measures

${ }^{\#}$ The multivariate effect of type of risk prime was not significant $(p=.094)$

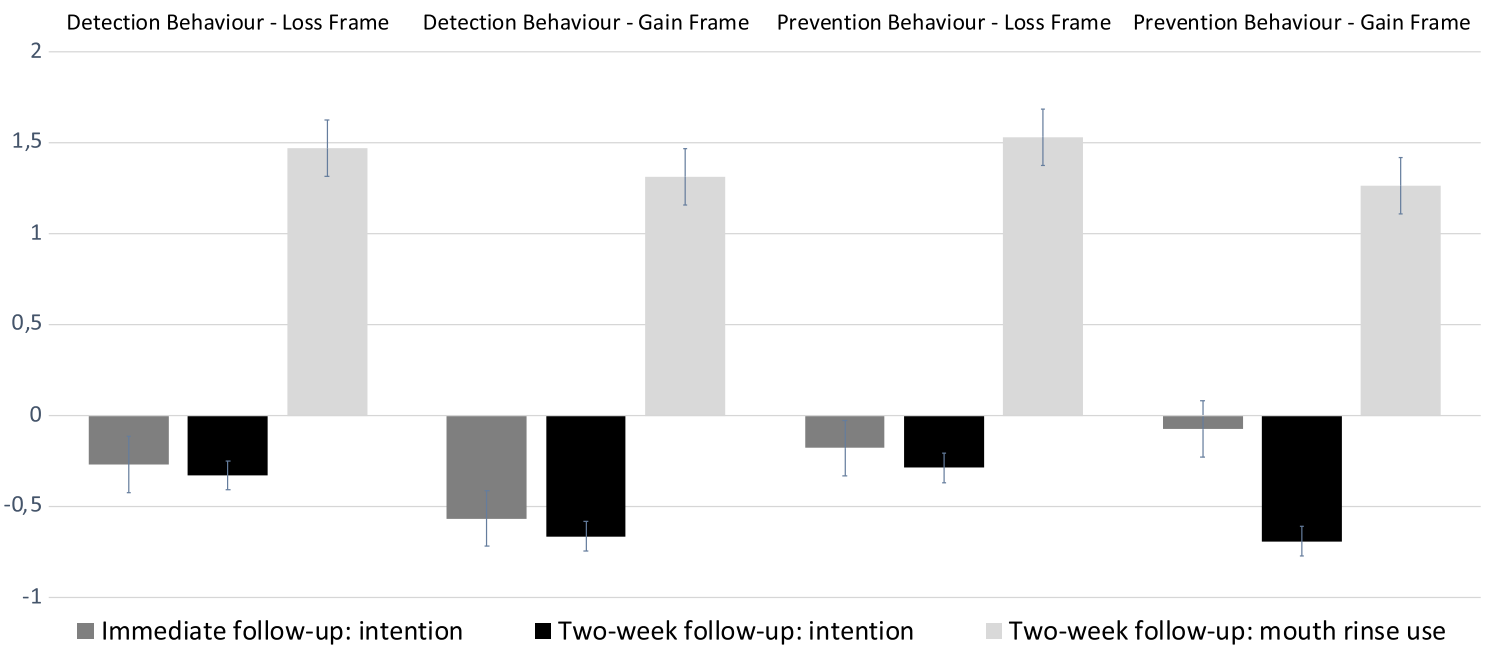

Fig. 3 Mean scores for dependent variables for the behavioral function x message frame interaction. Note Scores for intention could range from -3 (definitely not intending) to +3 (definitely intending). Scores for mouth rinse could range from 0 to 7 (days per week) 


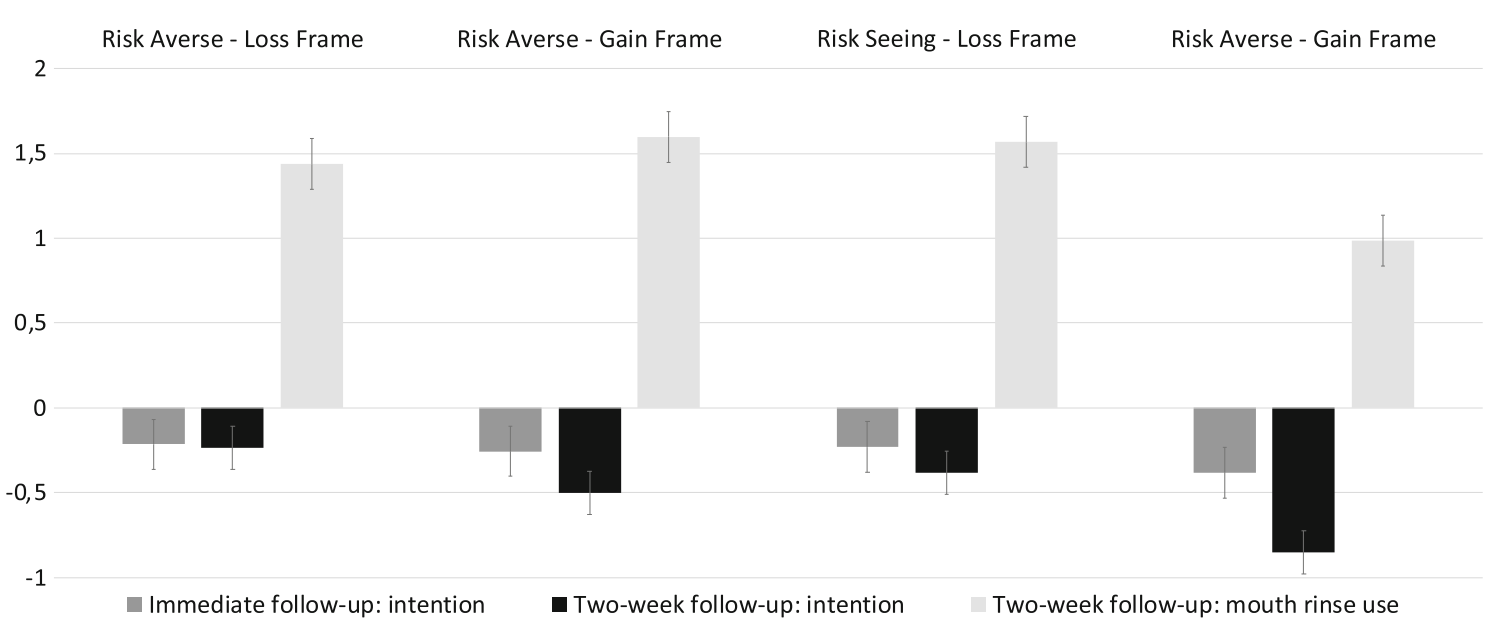

Fig. 4 Mean scores for dependent variables for the risk priming $\mathrm{x}$ message frame interaction. Note Scores for intention could range from -3 (definitely not intending) to +3 (definitely intending). Scores for mouth rinse could range from 0 to 7 (days per week)

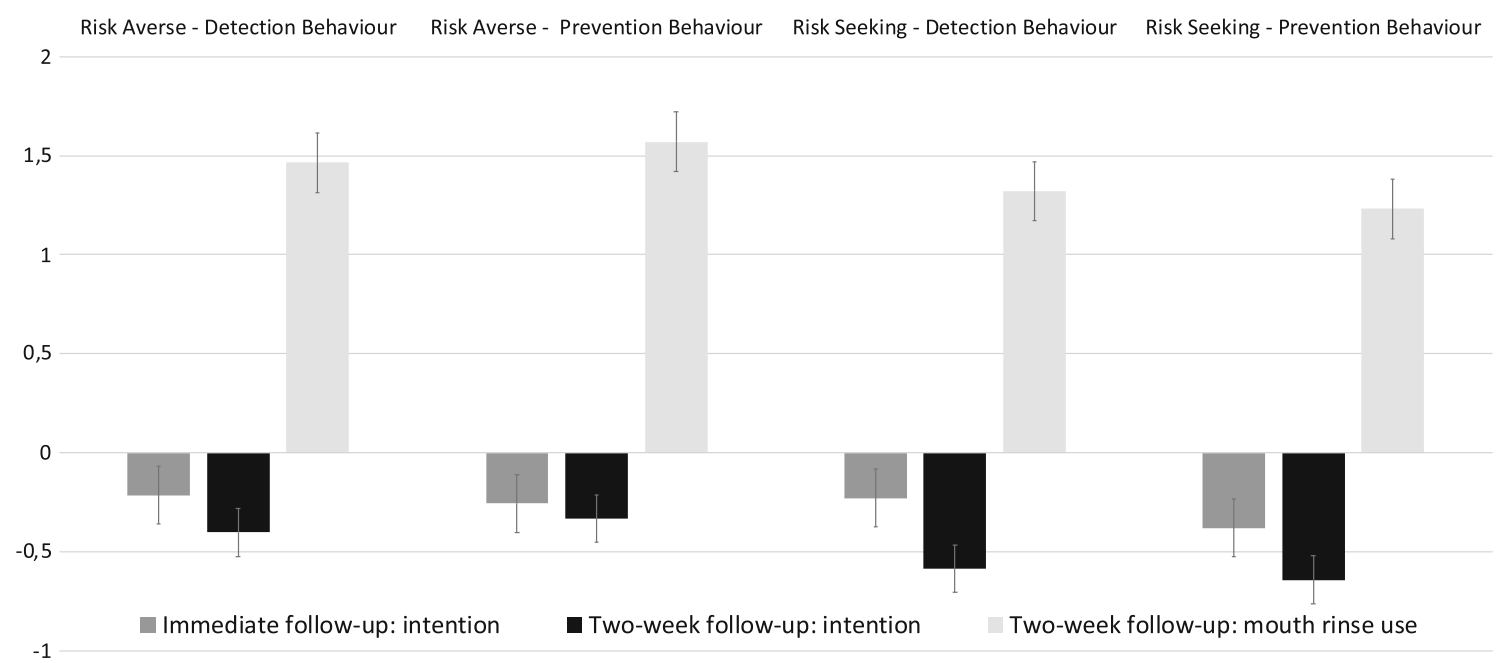

Fig. 5 Mean scores for dependent variables for the risk priming $\mathrm{x}$ behavioural function interaction. Note Scores for intention could range from -3 (definitely not intending) to +3 (definitely intending). Scores for mouth rinse could range from 0 to 7 (days per week)

framing theory. There was some support for the predicted interaction between message frame and behavioural function, with participants more likely to select a mouth rinse product that had a preventive function when that prevention function message emphasized gain-framed information rather than when it emphasised loss-framed information. However, the selection of a mouth rinse product with a detection function was no more likely when that detection function message emphasized loss-framed message than when it emphasized gain-framed information. Moreover, there was no interaction between behavioural function and message frame for the intention and the 2-week behavioural outcomes measures.

Overall, these findings lend some support for the tenets of message framing theory, but also highlight some limi- tations of these tenets. First, message framing predictions are more likely to be upheld when dichotomous choices are studied, as opposed to the more linear/interval scaling of standard intention and behavioural measures. This reflects earlier meta-analytical work that has often failed to find support for behavioural function* message frame interaction for interval measurement scales (Gallagher \& Updegraff, 2012) and in line with an overview study on message framing effects across different response modes (Kühberger, 1998). The present study extends these overview findings by applying it to a (dental) health problem and by investigating message framing effects on different response modes within the same sample. Second, the present study also suggests that the predicted interaction between message frame and behavioural function is more 
likely to be found for prevention behaviours than for detection behaviours. This also reflects earlier meta-analytical evidence where persuasive effects for prevention behaviours tend to be stronger than for detection behaviours (O'Keefe \& Jensen, 2006).

There was also some support for the interaction between message frame and the priming of risk. Although there were no main effects of message frame on mouth rinse use 2 weeks after experimental manipulation, participants who were primed to be risk-seeking had rinsed significantly more at the 2-week follow-up when they had read the lossframed information than when they had read the gainframed information. These findings indicate that the subliminal priming of a risk-seeking mind-set makes lossframed messages more persuasive, which is in accordance with earlier evidence applying self-reported measures of dental health risk (Updegraff et al., 2015). Although the present study used an elaborate risk priming task to mask the true purpose of the experimental setup, priming is commonly an easy to administer and unobtrusive strategy (Bargh \& Chartrand, 2000; Papies, 2016; Papies \& Hamstra, 2010) and should therefore be an efficient way to make loss-framed messages more persuasive in the dental health context.

The present study also revealed several inconsistent and mixed findings. For instance, there were inconsistent twoway interactions across the three outcome measures: immediate product choice was affected by the interaction between message frame and behavioural function, immediate intention was affected by the interaction between behavioural function and risk priming, and mouth rinse use at the 2-week follow-up was affected by the interaction between message frame and risk priming, with intention at the 2-week follow-up was affected by type of message frame only. Furthermore, although message frame and risk priming interacted in the prediction of mouth rinse use at the 2-week follow-up, the lack of three-way interaction also indicates that this effect is not moderated by the type of behavioural function (prevention or detection) detailed in the message. Finally, the interaction between message framing and risk priming also revealed that the differences between the two message frames was much less pronounced in the risk-avoidant priming conditions, while the decomposition of the interaction between message frame and behavioural function revealed only persuasive effects for gain-framed messages promoting mouth rinse with prevention functions. These inconsistencies are not unique to the present study and the lack of consistent evidence for message framing theory, both for main effects (Gallagher \& Updegraff, 2012; O'Keefe \& Jensen, 2006, 2009) and the presumed role of risk (Covey, 2015; Van 't Riet et al., 2014, 2016) has spurred debate on whether or not the message framing theory is a theoretically sound framework
(Van 't Riet et al., 2014) based on an insufficient translation of prospect theory tenets (Cox et al., 2006), and whether framing is a practically relevant persuasive communication strategy (Van 't Riet et al., 2016). The present study adds to this inconsistent body of evidence, despite a sufficiently powered sample, experimental manipulation of both behavioural function and message frame, and various persuasion outcomes. To completely understand how to successfully frame outcomes in order to maximize their persuasive effects and under which conditions and for which outcomes should remain a research priority in health communication.

A few limitations should be noted. First, mouth rinse use at the 2-week follow-up was assessed by self-report, which may not reflect actual use (Schwarz, 1999) and future research that uses more objective behavioural measures is needed to confirm the findings. Second, nearly twenty percent of participants were excluded from the analyses, because they did not regard the persuasive framed messages as intended. Although worrisome, this exclusion allows for a more certain conclusion of the intended persuasive effects because the included participants were not only more inclined to agree with the intended message frame, but also were more homogeneous in their perception. Additionally, this strategy has precedent in message framing studies in the dental hygiene domain (Rothman et al., 1999), but it does suggest that developing framed messages that are perceived by study participants as intended is a potential problem for message framing interventions. Likewise, it suggests that power analysis for message framing studies should potentially include a (standard) proportion value for loss due to failed manipulation. Third, an even larger proportion was excluded because the time they spent on the message page was too short. Although this may be a problem specific to online panel studies, most framing research has often failed to assess if and how people process and attend framed messages, suggesting that it should also be a research priority how people process and attend these messages, for instance via eye-tracking techniques (O' Malley \& Latimer, 2014). Finally, most research on risk and message framing have assessed behaviour-specific assessments of risk (e.g. If I do not engage in behaviour $\mathrm{X}$, I will risk negative consequences), but the present study used a priming task to induce either a general non-behaviour specific risk-seeking or a risk-averse mindset. Thus, the lack of risk moderation may also be due to the fact that the risk-priming task and the message content were not aligned in terms of specific behaviours. One way to overcome this would be to trigger a behaviour-specific risk-averse or risk-seeking mindset, for instance by having participants write down personal experiences about low or high risk situations regarding (non) use of mouth rinse (Pavey \& Sparks, 2012). It should 
also be noted that risk was assessed following theoretical guidelines for severity and susceptibility assessment (i.e. linking inaction to negative consequences), whereas some message framing work on risk has employed items assessing only consequences, such as the risk of having fillings or gum disease (Updegraff et al., 2015) or the negative consequences of action, such as discomfort or higher odds of unnecessary surgery of screening behaviours (Gallagher et al., 2011). Clearly, more consistent assessments are needed to fully understand how risk affects message framing effects.

Despite the limitations and the inconsistent findings, the present study has various strengths, including multiple outcome measures, manipulation of risk, function, and message frame, and a sufficiently powered sample. Practically speaking, the present findings indicate that mouth rinse use (as a frequency measure) can be successfully enhanced by pairing loss-framed information with the priming of a risk-seeking mind-set, whilst preventive mouth rinse product choice can be influenced by supplementing a prevention function with gain-framed information.
Acknowledgements The author wants to thank Bas van den Putte, Jonathan van 't Riet and John Updegraff for their useful comments during the design and drafting of this study.

\section{Compliance with ethical standards}

Conflict of interest Gert-Jan de Bruijn declares that he has no conflict of interest.

Human and animal rights and Informed consent All procedures were in accordance with the ethical standards of the institutional research committees and with the 1964 Helsinki declaration and its later amendments or comparable ethical standards. Informed consent was obtained from all individual participants included in the study.

Open Access This article is distributed under the terms of the Creative Commons Attribution 4.0 International License (http:// creativecommons.org/licenses/by/4.0/), which permits unrestricted use, distribution, and reproduction in any medium, provided you give appropriate credit to the original author(s) and the source, provide a link to the Creative Commons license, and indicate if changes were made.

\section{Appendix 1}

See Table 4.

Table 4 Sentences used in the scrambled sentence task

\begin{tabular}{|c|c|}
\hline Scrambled sentence & Correct sentence \\
\hline RS: Adventure is jumping daring parachute & Parachute jumping is daring \\
\hline RS: Dare are adventurous sports some & Some sports are adventurous \\
\hline RS: True risky can be diving & Diving can be risky \\
\hline RS: Basket insects fearlessly eat Sanne & Sanne eats insects fearlessly \\
\hline RS: Tim without casino gambles fear & Tim gambles without fear \\
\hline RS: Drivers Reckless where certain drive & Certain drivers drive reckless \\
\hline RS: Fierce handle risky is clothing & Fierce clothing is risky \\
\hline RS: Walk fearlessly second Mike at night & Mike walks fearless at night ${ }^{\mathrm{a}}$ \\
\hline RA: Vigilant dogs house are usually & Dogs are usually vigilant \\
\hline RA: Because are deeds her conscientious & Her deeds are conscientious \\
\hline RA: Thoughtful choices good makes Renee & Renee makes thoughtful choices ${ }^{b}$ \\
\hline RA: Ask Tamar nods pensively after & After, Tamar nods pensively \\
\hline RA: Viewers are observant work those & Those viewers are observant \\
\hline RA: Responsible I myself live feel & I feel myself responsible \\
\hline RA: Decisions informed balance makes Jesse & Jesse makes informed decisions \\
\hline RA: Cautious cat is the stairs & The cat is cautious \\
\hline FI: Book read top a they ${ }^{c}$ & They read a book ${ }^{\mathrm{d}}$ \\
\hline FI: I often Coke drink but ${ }^{\mathrm{c}}$ & I often drink Coke ${ }^{\mathrm{d}}$ \\
\hline FI: Bright yes light the shines ${ }^{c}$ & The light shines bright $^{\mathrm{d}}$ \\
\hline FI: The sweet tastes apple go & The apple tastes sweet ${ }^{\mathrm{d}}$ \\
\hline FI: Hot the opposite coffee is ${ }^{c}$ & The coffee is hot \\
\hline
\end{tabular}

$R S$ risk seeking, $R A$ risk avoidant, $F I$ filler

${ }^{\mathrm{a}}$ At night is one word in Dutch

'The Dutch translation of 'good' prevents the creation of a grammatically correct 'Renee makes good choices'

${ }^{c}$ Filler used in Risk Seeking condition

${ }^{\mathrm{d}}$ Filler used in the Risk Averse condition 


\section{Appendix 2: Persuasive messages}

\section{Gain frame: detection}

\section{Why mouth rinse? The benefits of healthy teeth}

Oral hygiene is important for many people. Detecting dental plaque, cavities and gum disease means you assess the quality of your teeth on time.

A detection mouth rinse will help you detect dental plaque, cavities, and gingivitis. This is a mouth rinse that you use after brushing your teeth. You take a little sip, rinse it through your mouth and spit it out afterwards. The rinse will leave a red layer on teeth and areas of your teeth where plaque, gingivitis and/or cavities are formed. This allows you to detect which areas you need to brush better. The layer disappears again after rinsing with (tap) water.

People who use a detection mouth rinse after they have brushed their teeth, have a bigger chance to detect, in a safe and effective way, areas in their teeth where plaque, cavities and gum disease have nestled. This allows them to identify areas that need better brushing. As a result, they have an increased chance to detect plaque, cavities and gum disease in time.

By using a detection mouth rinse, you will not only be able to assess the quality of your teeth on time, but you will also have a fresher breath, feel more confident about your teeth and gums, and increase the chance to keep your own teeth.

\section{Loss frame: detection}

\section{Why mouth rinse? The cons of unhealthy teeth}

Oral hygiene is important for many people. Not detecting dental plaque, cavities and gum disease means you cannot assess the quality of your teeth on time.

A detection mouth rinse will help you detect dental plaque, cavities, and gingivitis. This is a mouth rinse that you use after brushing your teeth. You take a little sip, rinse it through your mouth and spit it out afterwards. The rinse will leave a red layer on teeth and areas of your teeth where plaque, gingivitis and/or cavities are formed. This allows you to detect which areas you need to brush better. The layer disappears again after rinsing with (tap) water.

People who do not use a detection mouth rinse after they have brushed their teeth, have a smaller chance to detect, in a safe and effective way, areas in their teeth where plaque, cavities and gum disease have nestled. This allows them to identify areas that need better brushing. As a result, they have a decreased chance to detect plaque, cavities and gum disease in time. By not using a detection mouth rinse, you will not only be able to assess the quality of your teeth too late, but you will also have a less fresher breath, feel less confident about your teeth and gums, and decrease the chance to keep your own teeth.

\section{Gain frame: prevention}

\section{Why mouth rinse? The benefits of healthy teeth}

Oral hygiene is important for many people. Preventing dental plaque, cavities and gum disease means the quality of your teeth will be maintained.

A prevention mouth rinse will help you prevent dental plaque, cavities, and gingivitis. This is a mouth rinse that you use after brushing your teeth. You take a little sip, rinse it through your mouth and spit it out afterwards. The rinse will neutralize bacteria that are the main cause of plaque, gingivitis and/or cavities are formed.

People who use a prevention mouth rinse after they have brushed their teeth, have a bigger chance to tackle areas in their teeth where plaque, cavities and gum disease have nestled in a safe and effective way. As a result, they have an increased chance to prevent plaque, cavities and gum disease in time.

By using a prevention mouth rinse, you will not only be able to maintain healthy teeth, but you will also have a fresher breath, feel more confident about your teeth and gums, and increase the chance to keep your own teeth.

\section{Loss frame: prevention}

\section{Why mouth rinse? The cons of unhealthy teeth}

Oral hygiene is important for many people. Not preventing dental plaque, cavities and gum disease means the quality of your teeth will deteriorate.

A prevention mouth rinse will help you prevent dental plaque, cavities, and gingivitis. This is a mouth rinse that you use after brushing your teeth. You take a little sip, rinse it through your mouth and spit it out afterwards. The rinse will neutralize bacteria that are the main cause of plaque, gingivitis and/or cavities are formed.

People who do not use a prevention mouth rinse after they have brushed their teeth, have a smaller chance to tackle areas in their teeth where plaque, cavities and gum disease have nestled in a safe and effective way. As a result, they have an increased chance that plaque, cavities and gum disease will not prevented in time.

By not using a prevention mouth rinse, you will not only be able to not maintain healthy teeth, but you will also have a less fresher breath, feel less confident about your teeth and gums, and decrease the chance to keep your own teeth. 


\section{References}

Allen, P. F. (2003). Assessment of oral health related quality of life. Health and Quality of Life outcomes, 1, 40.

Bargh, J. A., \& Chartrand, T. L. (2000). The mind in the middle: A practical guide to priming and automaticity research. In $\mathrm{H}$. T. Reis \& C. M. Judd (Eds.), Handbook of research methods in social psychology (pp. 253-285). New York: Cambridge University Press.

Chen, H., Cohen, P., \& Chen, S. (2010). How big is a big odds ratio? Interpreting the magnitudes of odds ratios in epidemiological studies. Communications in Statistics - Simulation and Computation, 39(4), 860-864. https://doi.org/10.1080/ 03610911003650383

Cohen, J. (1992). A power primer. Psychological Bulletin, 112, $155-159$.

Covey, J. (2015). The role of dispositional factors in moderating message framing effects. Health Psychology, 33, 52-65. https:// doi.org/10.1037/a0029305

Cox, A. D., Cox, D., \& Zimet, G. (2006). Understanding consumer responses to product risk information. Journal of Marketing, 70, 79-91.

Erb, H.-P., Bioy, A., \& Hilton, D. J. (2002). Choice preferences without inferences: Subconscious priming of risk attitudes. Journal of Behavioral Decision Making, 15, 251-262. https:// doi.org/10.1002/bdm.416

Faul, F., Erdfelder, E., Lang, A.-G., \& Buchner, A. (2007). G* Power 3: A flexible statistical power analysis program for the social, behavioral, and biomedical sciences. Behavior Research Methods, 39, 175-191.

Gallagher, K. M., \& Updegraff, J. A. (2012). Health message framing effects on attitudes, intentions, and behavior: A meta-analytic review. Annals of Behavioral Medicine, 43, 101-116. https://doi. org/10.1007/s12160-011-9308-7

Gallagher, K. M., Updegraff, J. A., Rothman, A. J., \& Sims, L. (2011). Perceived susceptibility to breast cancer moderates the effect of gain- and loss-framed messages on use of screening mammography. Health Psychology, 30, 145-152. https://doi.org/ 10.1037/a0022264

Gilad, D., \& Kliger, D. (2008). Priming the risk attitudes of professionals in financial decision making. Review of Finance, 12, 567-586. https://doi.org/10.1093/rof/rfm034

Griffin, S. O., Jones, J. A., \& Brunson, D. (2012). Burden of oral disease among older adults and implications for public health priorities. Journal of Public Health, 102, 411-418.

Henderson, C. J., Hagger, M. S., \& Orbell, S. (2007). Does priming a specific illness schema result in an attentional informationprocessing bias for specific illnesses? Health Psychology, 26, 165-173. https://doi.org/10.1037/0278-6133.26.2.165

Herr, P. M. (1986). Consequences of priming: Judgment and behavior. Journal of Personality and Social Psychology, 51, 1106-1115. https://doi.org/10.1037/0022-3514.51.6.1106

Janiszewski, C., \& Wyer, R. S. (2014). Content and process priming: A review. Journal of Consumer Psychology, 24, 96-118. https:// doi.org/10.1016/j.jcps.2013.05.006

Janz, N. K., \& Becker, M. H. (1984). The health belief model: A decade later. Health Education \& Behavior, 11, 1-47.

Kahneman, D., \& Tversky, A. (1979). Prospect theory: An analysis of decision under risk. Econometrica: Journal of the Econometric Society, 47, 263-291.

Kühberger, A. (1998). The influence of framing on risky decisions: A meta-analysis. Organizational Behavior and Human Decision Processes, 75, 23-55.

Li, K.-K., Cheng, S.-T., \& Fung, H. H. (2014). Effects of message framing on self-report and accelerometer-assessed physical activity across age and gender groups. Journal of Sport and Exercise Psychology, 36, 40-51. https://doi.org/10.1123/jsep. 2012-0278

Mann, T., Sherman, D., \& Updegraff, J. (2004). Dispositional motivations and message framing: A test of the congruency hypothesis in college students. Health Psychology, 23, 330-334. https://doi.org/10.1037/0278-6133.23.3.330

Marinho, V. C., Chong, L. Y., Worthington, H. V., \& Walsh, T. (2016). Fluoride mouthrinses for preventing dental caries in children and adolescents. Cochrane Database of Systematic Reviews. https://doi.org/10.1002/14651858.CD002284.pub2

Mattila, K. J., Nieminen, M. S., Valtonen, V. V., \& Rasi, V. P. (1989). Association between dental health and acute myocardial infarction. British Medical Journal, 298, 779.

O' Malley, D., \& Latimer, A. (2014). Gaining perspective: The effects of message frame on viewer attention to and recall of osteoporosis prevention print advertisements. Journal of Health Psychology, 18, 1400-1410. https://doi.org/10.1177/ 1359105312456323

O'Keefe, D. J., \& Jensen, J. D. (2006). The advantages of compliance or the disadvantages of noncompliance? A meta-analytic review of the relative persuasive effectiveness of gain-framed and lossframed messages. Annals of the International Communication Association, 30, 1-43. https://doi.org/10.1080/23808985.2006. 11679054

O'Keefe, D. J., \& Jensen, J. D. (2009). The relative persuasiveness of gain-framed and loss-framed messages for encouraging disease detection behaviors: A meta-analytic review. Journal of Communication, 59, 296-316. https://doi.org/10.1111/j.1460-2466. 2009.01417.x

Pakpour, A. H., Yekaninejad, M. S., Sniehotta, F. F., Updegraff, J. A., \& Dombrowski, S. U. (2013). The effectiveness of gain- versus loss-framed health messages in improving oral health in Iranian secondary schools: A cluster-randomized controlled trial. Annals of Behavioral Medicine, 47, 376-387. https://doi.org/10.1007/ s12160-013-9543-1

Papies, E. K. (2016). Health goal priming as a situated intervention tool: How to benefit from nonconscious motivational routes to health behaviour. Health Psychology Review, 10, 408-424. https://doi.org/10.1080/17437199.2016.1183506

Papies, E. K., \& Hamstra, P. (2010). Goal priming and eating behavior: Enhancing self-regulation by environmental cues. Health Psychology, 29, 384-388. https://doi.org/10.1037/ a0019877

Pavey, L. J., \& Sparks, P. (2012). Autonomy and defensiveness: Experimentally increasing adaptive responses to health-risk information via priming and self-affirmation. Psychology \& Health, 27, 259-276. https://doi.org/10.1080/08870446.2011. 556251

Rogers, R. W., \& Prentice-Dunn, S. (1997). Protection motivation theory. In D. S. Gochman (Ed.), Handbook of health behavior research 1: Personal and social determinants (pp. 113-132). New York: Plenum Press.

Rothman, A. J., Martino, S. C., Bedell, B. T., Detweiler, J. B., \& Salovey, P. (1999). The systematic influence of gain-and lossframed messages on interest in and use of different types of health behavior. Personality and Social Psychology Bulletin, 25, 1355-1369.

Rothman, A. J., \& Salovey, P. (1997). Shaping perceptions to motivate healthy behavior: The role of message framing. Psychological Bulletin, 121, 3-19.

Schwarz, N. (1999). Self-reports: How the questions shape the answers. American Psychologist, 54, 93-105.

Sheiham, A. (2005). Oral health, general health and quality of life. Bulletin of the World Health Organization, 83, 644. 
Tonetti, M. S., Eickholz, P., Loos, B. G., Papapanou, P., van der Velden, U., Armitage, G., et al. (2015). Principles in prevention of periodontal diseases: Consensus report of group 1 of the 11th European Workshop on Periodontology on effective prevention of periodontal and peri-implant diseases. Journal of Clinical Periodontology, 42, S5-11. https://doi.org/10.1111/jcpe.12368

Updegraff, J. A., Brick, C., Emanuel, A. S., Mintzer, R. E., \& Sherman, D. K. (2015). Message framing for health: Moderation by perceived susceptibility and motivational orientation in a diverse sample of Americans. Health Psychology, 34, 20-29. https://doi.org/10.1037/hea0000101

Updegraff, J. A., Sherman, D. K., Luyster, F. S., \& Mann, T. L. (2007). The effects of message quality and congruency on perceptions of tailored health communications. Journal of Experimental Social Psychology, 43, 249-257. https://doi.org/ 10.1016/j.jesp.2006.01.007

Van 't Riet, J., Cox, A. D., Cox, D., Zimet, G. D., De Bruijn, G.-J., Van den Putte, B., et al. (2014). Does perceived risk influence the effects of message framing? A new investigation of a widely held notion. Psychology \& Health, 29, 933-949. https://doi.org/ 10.1080/08870446.2014.896916
Van 't Riet, J., Cox, A. D., Cox, D., Zimet, G. D., De Bruijn, G. J., Van den Putte, B., et al. (2016). Does perceived risk influence the effects of message framing? Revisiting the link between prospect theory and message framing. Health Psychology Review, 10, 447-459. https://doi.org/10.1080/17437199.2016. 1176865

Van Leeuwen, M., Rosema, N., Versteeg, P. A., Slot, D. E., Hennequin-Hoenderdos, N. L., \& Van der Weijden, G. A. (2017). Effectiveness of various interventions on maintenance of gingival health during 1 year-A randomized clinical trial. International Journal of Dental Hygiene, 15, e16-e27. https:// doi.org/10.1111/idh.12213

Weinstein, N. D. (1987). Unrealistic optimism about susceptibility to health problems: Conclusions from a community-wide sample. Journal of Behavioral Medicine, 10, 481-500.

Weinstein, N. D., \& Klein, W. M. (1995). Resistance of personal risk perceptions to debiasing interventions. Health Psychology, 14, 132-140. 